\title{
Fuzzy logic-based modelling of yield strength of as-cast A356 alloy
}

\author{
Vladimir Tarasov $^{1}$ (D) He $\operatorname{Tan}^{1} \cdot$ Anders E. W. Jarfors $^{2} \cdot$ Salem Seifeddine $^{2}$
}

Received: 13 July 2018/Accepted: 23 January 2019/Published online: 7 February 2019

(C) The Author(s) 2019

\begin{abstract}
Uncertain and imprecise data are inherent to many domains, e.g. casting lightweight components. Fuzzy logic offers a way to handle such data, which makes it possible to create predictive models even with small and imprecise data sets. Modelling of cast components under fatigue load leads to understanding of material behaviour on component level. Such understanding is important for the design for minimum warranty risk and maximum weight reduction of lightweight cast components. This paper contributes with a fuzzy logic-based approach to model fatigue-related mechanical properties of as-cast components, which has not been fully addressed by the current research. Two fuzzy logic models are constructed to map yield strength to the chemical composition and the rate of solidification of castings for two A356 alloys. Artificial neural networks are created for the same data sets and then compared to the fuzzy logic approach. The comparison shows that although the neural networks yield similar prediction accuracy, they are less suitable for the domain because they are opaque models. The prediction errors exhibited by the fuzzy logic models are 3.53\% for the model and 3.19\% for the second, which is the same error level as reported in related work. An examination of prediction errors indicated that these are affected by parameters of the membership functions of the fuzzy logic model.
\end{abstract}

Keywords Fuzzy logic · Membership functions · Artificial neural networks · Prediction accuracy · Mechanical properties prediction · A356 alloy · Cast components

\section{Introduction}

Uncertain and imprecise data are inherent to complex systems. To effectively model uncertainty and imprecision, performance and quality of raw data must be understood

The research reported in this paper has been financed by Grant \#20170066 from the Knowledge Foundation (Sweden).

Vladimir Tarasov

vladimir.tarasov@ju.se

He Tan

he.tan@ju.se

Anders E. W. Jarfors

anders.jarfors@ju.se

Salem Seifeddine

salem.seifeddine@ju.se

1 Department of Computer Science and Informatics, School of Engineering, Jönköping University, Box 1026,

55111 Jönköping, Sweden

2 Department of Materials and Manufacturing, School of Engineering, Jönköping University, Box 1026, 55111 Jönköping, Sweden and managed. In addition to this, not all data sets will be complete and data may exist only in discrete ranges. Fuzzy logic has been proposed as a method to deal with uncertain, imprecise and incomplete data [34]. The engineering domains provide many examples of fuzzy logic applications dealing with uncertainty and imprecision. Bed-to-wall heat transfer in circulating fluidised bed boilers has been modelled with fuzzy logic in $[17,18]$. The constructed model was used to predict the overall heat transfer coefficient in the combustion chamber with the maximum relative prediction error lower than 3\%. A comparison of fuzzy logic with a more traditional cluster renewal approach [3] showed that the fuzzy logic model exhibits the same accuracy but at the same time provides an advantage of simple investigation of the influence of input parameters on the output values. Fuzzy logic models have been created to model the pullout strengths of near-surface-mounted fibrereinforced polymer strips or rods [22]. The results showed better accuracy of the constructed models than the published models as well as high interpretability of the fuzzy rule base of the Mamdani system. Among IT-related examples is determination of a learning level of a student with a fuzzy logic model that is part of an intelligent 
tutoring system [14]. The fuzzy logic decision system helps to eliminate uncertainty in the student's score that enhance individual learning. Fuzzy logic has also been used to detect selfish nodes in ad hoc networks [11]. The results showed that the fuzzy logic approach performs better than deterministic algorithms for determining node status.

Casting is an important engineering area, which provides very effective methods to produce near net shape components and offers a great design freedom [13]. For lightweight cast components, the automotive industry is the largest user. ${ }^{1}$ This will be strongly affected by the transition from the use of combustion engines to electrical vehicles where lightweight design and sustainability are likely to increase in importance. Designing for weight reduction can be made by material replacement or by a lighter design in similar materials. Most commonly, this means less material to carry the load resulting in similar or higher levels of stress making it necessary to understand and to describe material behaviour on component level in detail to design for minimum warranty risk and maximum weight reduction. Most castings fail due to fatigue load but for automotive applications ductility requirements are equally important. The understanding of this behaviour is under extensive research for wrought materials, but cast materials, with many different large phases and great variation in mechanical properties, are poorly understood [16] and the spread in fatigue life is significant [28] as well as in ductility.

The performance of a cast component depends on several factors. Deterministic models will be highly useful in a part design process as it will be distinct differences between different design and solutions. It must be pointed out, however, that other imperfections resulting from melt treatment and casting process will inherently display a stochastic behaviour. In addition to this, there are a number of uncontrolled parameters that vary in a foundry, which will also result in variations in part quality and accuracy of a simulation [25]. These variations will limit the ability of a deterministic model to predict the actual outcome as well as necessitate management of the uncertainty. The quality of available data also affects prediction capabilities. For example, material performance of a part shows inherent variations due to the location of the tensile bar and variations from different foundries producing the casting [29]. Data produced in this way indicate part performance but are rather imprecise. The alloy type varies as well, and the variation may be confined within one specific specification that for instance after heat treatment of $\mathrm{AlSi} 9 \mathrm{Cu} 3 \mathrm{Fe}$ could be reduced with one-third compared to the peek value [20].

\footnotetext{
${ }^{1}$ OICAs statistics of worldwide vehicle sales http://www.oica.net/ category/sales-statistics/.
}

Industrial applications of fuzzy control, which are based on fuzzy logic, have been successful in managing complex nonlinear systems [24]. In particular, fuzzy logic models have been used to predict mechanical properties such as hardness and roughness of cast and metallic materials [2, 12, 27, 35]. However, the current research has not fully addressed mapping of mechanical properties of metallic materials, which are related to fatigue stress, to the process parameters. This paper investigates the use of fuzzy logic systems for the prediction of mechanical properties of ascast components based on experimental data. The fuzzy logic models are compared with artificial neural networks (ANN) created for the same data. The main contributions of the paper are (1) to propose fuzzy logic models for prediction of yield strength of as-cast components with satisfactory accuracy, and (2) to show that the performance of fuzzy logic modelling is the same or better compared to ANN in the case of small experimental data sets. This initial work is delimited to an as-cast A356 alloy type of chemistry and microstructure.

The paper is structured as follows. Section 2 introduces fuzzy logic systems and describes related work. Section 3 presents details of melt preparation and casting, and results of tensile testing. The proposed approach to fuzzy logic modelling is described in Sect. 4. The evaluation of the constructed models is presented in Sect. 5. The comparison of the fuzzy logic approach to the use of artificial neural networks for prediction is described in Sect. 6. The results are discussed in Sect. 7. The conclusions are drawn in Sect. 8.

\section{Related work}

\subsection{Handling uncertainty when modelling ductile failures}

Models of mechanical properties of cast and metallic materials are often constructed with the help of empirical data. Working with such data that are collected through laboratory tests and filed experiments involves handling uncertainty. Probabilistic methods are powerful tools to deal with uncertainties. They have been applied to predict component performance since the Weibull distribution was proposed in 1951, representing the distribution of steel yield strength and fatigue life [31]. In contrast to deterministic analysis, a probabilistic method represents the input parameters as probability distributions and identifies the distributions of performance. Additionally, as part of the analysis, the most critical input parameters to the performance distribution can be identified. Various studies corroborate this by successful investigations of different probabilistic aspects of component performance, including 
variability in stress-life data (e.g. [5, 19]), fatigue crack growth rate (e.g. [7, 32]), life prediction considering microstructure (e.g. [26, 30]), etc.

Probabilistic methods handle uncertainty in the form of probability based on bivalent logic [33]. Every proposition is either true or false, with no shades of grey allowed. The probability of a proposition is the degree of belief on the truth of the proposition. On the contrary, fuzzy logic is a logic of imprecise propositions that may be more or less true. The truth of a proposition is a matter of degree. Fuzzy logic deals with uncertainty and imprecision of which both are inherent parts of complex systems. This makes a fuzzy logic approach very interesting for the prediction of ductile failure. Hence, fuzzy logic is an appropriate approach to predict cast component performance, particularly, fatigue life properties for ductile materials to which probabilistic methods are less likely to be successful [8].

\subsection{Application of fuzzy logic to model mechanical properties of industrial materials}

Fuzzy logic has long been applied to industrial problems with successful results [24]. However, very few studies have applied fuzzy logic in casting and metal industry. Several papers have been published on predicting surface roughness and hardness of cast and metallic components. The authors in [2] used fuzzy logic to predict surface roughness of die casting alloys after machining. The $\mathrm{Al}-$ $11.3 \mathrm{Si}-2 \mathrm{Cu}-0.4 \mathrm{Fe}$ alloy was treated with different additives such as $\mathrm{Sr}, \mathrm{Bi}$, and $\mathrm{Sb}$, and workpieces were cast after that. The surface roughness of a workpiece was measured after the machining. The fuzzy logic modelling resulted in mapping the presence of additives and machining parameters to surface roughness with the error of $5.4 \%$. In the work [12], a fuzzy expert system is described that first optimises parameters of the milling process for a given objective, e.g. maximise tool life, and then infers predicted values of performance measures for the selected milling parameters. The fuzzy logic module of the expert system is used to predict surface roughness of a machined workpiece based on milling orientation, cutter's helix angle, workpiece material hardness and coolant.

Surface hardness of aluminium alloy with TiN coating has been predicted in [35] with the error of $3.85 \%$. The TiN (titanium nitride) coating was added on Al-7075-T6 by a sputtering machine, and then the surface hardness was measured. The fuzzy logic model built by the authors predicts surface hardness with the help of four inputs: direct current power, substrate temperature, nitrogen flow rate and direct current bias voltage. The work [27] describes the application of a neuro-fuzzy system and particle swarm optimisation to determine optimal process parameters for aluminium metal matrix composites. In the study, a A356 matrix was reinforced with $\mathrm{Al}_{2} \mathrm{O}_{3}$ nanoparticles before casting and tension tests were performed. The adaptive neuro-fuzzy inference system built by the authors can estimate hardness of a compo casting with the average testing error of 3.96. The neuro-fuzzy system uses seven inputs: mould temperature, mix time, impeller speed, powder temperature, cast temperature, particle volume percentage and particle size.

Moreover, fuzzy logic has been used to predict mechanical properties of non-metallic materials or to relate resulting mechanical properties to the casting process. The work in [4] aimed at predicting mechanical properties of cross-linked polyethylene (XLPE) insulation used in medium- and high-voltage cables. The insulation samples were aged at four different temperatures and then subjected to tensile stress. The built fuzzy logic model maps the ageing time and ageing temperature to tensile strength, elongation at rupture and hot set test elongation. The relative error of the model is between 1.6 and $5.0 \%$. The authors in [6] have used fuzzy logic in combination with grey relational analysis to determine optimal parameters for die casting of AZ91D magnesium alloy components. The resulting mechanical properties, namely casting density, warpage and flow mark were first transformed into grey relational coefficients based on the experimental data. After that, the fuzzy logic module computed grey-fuzzy reasoning grade, which was used to find optimal casting parameters: die temperature, the pressure of injection, the plunger velocity and the filling time. The results showed improvements of the die casting process by using the greybased fuzzy approach.

Hence, fuzzy logic has been applied to model mechanical properties of die casts and metallic materials with good accuracy. The predicted properties were surface roughness and hardness. Fuzzy logic was also successfully used to predict tensile strengths and elongation at rapture of insulation material. However, there are only limited attempts to predict mechanical properties, such as yield strength, of cast components, which is the focus of this paper and then in particular the aluminium alloy A356 and linking the performance to process and microstructural in-data.

\subsection{Artificial neural networks for prediction of mechanical properties of alloys}

Artificial neural networks (ANN) are a widely accepted method to deal with complex and nonlinear problems in an alternative way. Once trained, ANN can perform prediction and generalisation at high speed and with good accuracy. Many applications of ANN can be found in modelling and predicting mechanical properties of industry materials (e.g. $[10,21,23])$. The work that is most relevant to this paper is the one by Ozerdem and Kolukisa published in [23]. They 
applied an ANN approach to predict the tensile strength, yield strength and elongation of $\mathrm{Cu}-\mathrm{Sn}-\mathrm{Pb}-\mathrm{Zn}-\mathrm{Ni}$ cast alloys given $\mathrm{Cu}-\mathrm{Sn}-\mathrm{Pb}-\mathrm{Zn}-\mathrm{Ni}$ (wt.\%) contents as the input. The intent of the presented work is to apply the fuzzy logic approach to predict mechanical properties of as-cast alloys as an alternative method. In contrast to an ANN that is an opaque model, "black box", a fuzzy logic model is a "white box". This makes the fuzzy logic approach especially interesting for industrial applications as soon as model transparency is often required by the stakeholders for the model to be used at a working place. The advantage of the transparency of the fuzzy system is pointed out in [22]. In the presented paper, ANN models were created for the same prediction task. The fuzzy logic approach has been evaluated against the ANN approach to demonstrate that both approaches yield very similar predication quality.

\section{Experimental work}

This section first describes the process to cast cylindrical rods. After that, the procedure is presented to test bars machined from the solidified rods. The data collected during this experimental phase were used to create the fuzzy logic models presented in Sect. 4.

\subsection{Melt preparation and casting}

Seven $\mathrm{Al}-7 \% \mathrm{Si}-0.4 \% \mathrm{Mg}$ alloys, based on $\mathrm{A} 356$ master alloy, modified with approximately 200-250 ppm Sr and alloyed with various $\mathrm{Cu}$ concentrations were melt in a resistance furnace. Cylindrical rods (length $18 \mathrm{~cm}$, diameter $1 \mathrm{~cm}$ ) were thereafter cast in a $200{ }^{\circ} \mathrm{C}$ preheated permanent copper mould. Afterwards, the rods were inserted into the Bridgeman furnace, Fig. 1 , at $710{ }^{\circ} \mathrm{C}$, remelted for $20 \mathrm{~min}$. and thereafter directionally solidified. The speed of the furnace during the passage of molten rod during the cooling channels determines the solidification rate of the samples; different microstructures can thereby be produced by changing the speed. Three different coarsenesses of the microstructure having Secondary Dendrite Arm Spacing (SDAS) of approximately 10, 25 and $50 \mu \mathrm{m}$ were directionally solidified for the present investigation. Water cooling was used for high furnace speeds, $3 \mathrm{~mm} / \mathrm{s}$ and $0.3 \mathrm{~mm} / \mathrm{s}$ corresponding to SDAS of 10 and $25 \mu \mathrm{m}$, respectively, whereas no water cooling was used for the $0.03 \mathrm{~mm} / \mathrm{s}$ velocity that corresponds to SDAS of $50 \mu \mathrm{m}$; instead, air was the cooling media.

\subsection{Tensile testing}

Tensile test bars with a gauge length of $50 \mathrm{~mm}$ and a diameter of $7 \mathrm{~mm}$ were machined from the directionally

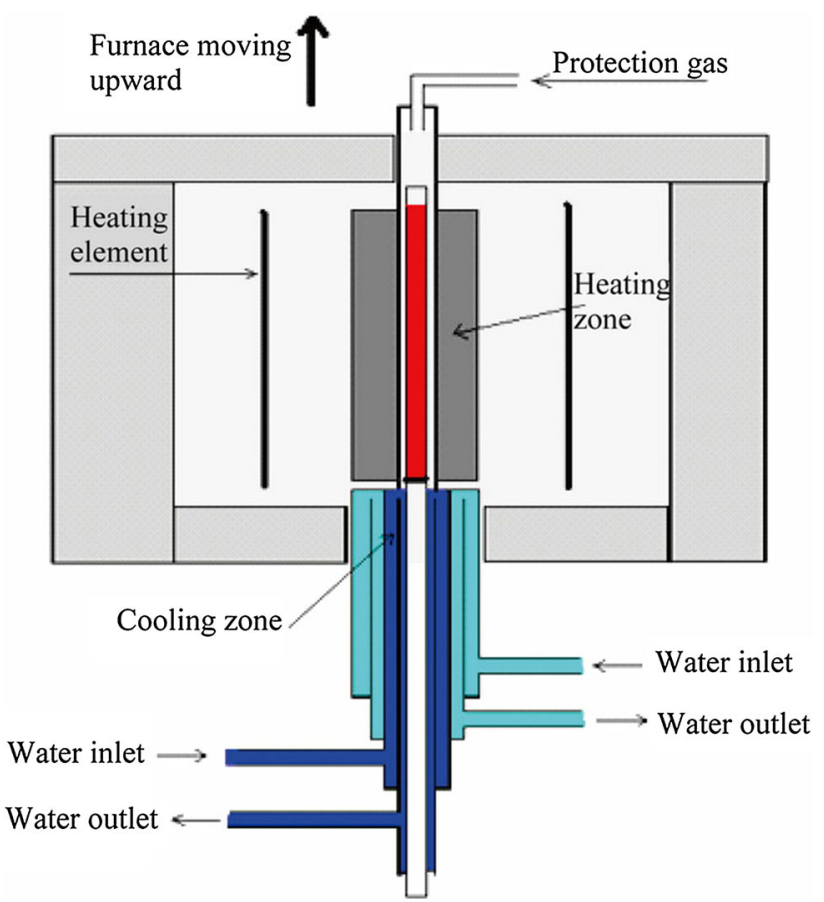

Fig. 1 The Bridgman furnace; directional solidification equipment

solidified rods. Tensile tests were performed at a constant strain rate of $0.5 \mathrm{~mm} / \mathrm{min}$ using a Zwick/Roell Z100 machine equipped with a 100-kN load cell and a clip-on 20 $\mathrm{mm}$ gauge length extensometer. The samples were tested until fracture, using three tensile test bars for each condition. Since the proposed directional solidification technology has proven to deliver optimal tensile test results due to excellent feeding during solidification, revealing the potential of studied alloys, only samples that represent the potential of the alloy in terms of tensile properties are presented.

\section{Fuzzy logic models to predict yield strength of cast components}

The fuzzy logic modelling presented in this section was carried out with the help of data obtained during the experimental work described in Sect. 3. As soon as two different additives, $\mathrm{Cu}$ and $\mathrm{Si}$, were used, two versions of a fuzzy inference system were created to predict yield strength of a cast component. The Matlab Fuzzy Logic Toolbox was used to implement the Mamdani fuzzy inference systems. The experimental results provided data on cast specimens that included variation in the casting process-SDAS - and variation in the chemical composition of cast alloys-percentage of $\mathrm{Cu}$ or $\mathrm{Si}$. As described in Sect. 3.1, the speed of the furnace during the passage of molten rod during the cooling channels was varied, which 
lead to different coarsenesses of the specimen microstructure measured as Secondary Dendrite Arm Spacing (SDAS). The experimental data also contained measurements of mechanical properties-yield strength in this study.

The first series of experiments was conducted on specimens cast with the alloy A356-Al-7\% $\mathrm{Si}-0.4 \%-\mathrm{Mg}-$ $0.25 \% \mathrm{Fe}$ with variation of $\mathrm{Cu}$. The second series was based on alloy $\mathrm{A} 356-\mathrm{Al}-7 \% \mathrm{Si}-0.4 \% \mathrm{Mg}$ with variation of $\mathrm{Si}$. In both series, the solidification rate of the samples was varied as described in Sect. 3.1. The first data set contained 67 data points. They were divided into two parts: a training data set with 46 data points and a test data set with 21 data points. The second data set contained 52 data points. This data set was divided into two parts: a training data set with 36 data points and a test data set with 16 data points. The complete data sets were used to define required membership functions for input and output linguistic variables (Sect. 4.1), while the training data sets were utilised to derive fuzzy inference rules (Sect. 4.2). The utilised aggregation method was max, and the defuzzification method was centroid. The prediction accuracy of the created fuzzy models was evaluated with the help of the test data sets (Sect. 5).

\subsection{Linguistic variables and membership functions}

The first phase of the construction of the fuzzy logic systems was to define linguistic variables. The required variables were identified after the training data sets inspection and discussion with domain experts. Two input variables were defined: "sdas", representing microstructure coarseness, and "Cu-amount" or "Si-amount", representing the percentage of $\mathrm{Cu}$ in the first data set and the percentage of $\mathrm{Si}$ in the second data set, respectively. One output variable "yield-strength" was defined for the resulting yield strength. The Gaussian type was chosen for the membership functions of all the variables for the following reasons. First, the domain experts considered the Gaussian type to be the most adequate based on the experimental data. Second, Gaussian and triangular are often used as the membership function types in similar domains $[2,4,12,27,35]$. The examination of the training data sets included two steps:

1. Compilation of the existing values of an input/output (a process parameter or mechanical property) in a list and sorting it to determine the range of the variable.

2. Partitioning of the value list into segments to determine the number of membership functions (linguistic labels) for a variable and the parameters for each membership function, i.e. $\sigma$, which controls the width of the "bell" of the curve, and $c$, which defines the position of the centre of the curve peak. The size of each partition was chosen to encompass a few values, and the overall partitioning was discussed with the domain experts.

The linguistic variables with their characteristics for the input and output parameters are presented in Table 1. The three membership functions of the first input variable for both experimental series correspond to the three coarsenesses of microstructure presented in Sect. 3.1. They are shown in Fig. 2c. The second input variable required five membership functions for both experimental series. Fig. 2a depicts the membership functions for the first data set and Fig. $2 \mathrm{~b}$ for the second data set. The amount of the additives ( $\mathrm{Cu}$ and $\mathrm{Si}$ ) was changed in uneven steps according to the judgement of the domain experts. As a result, the shapes of the membership functions are not uniform to allow for a finer distinction between lower percentages of $\mathrm{Cu}$ or higher percentages of Si that were more often used in the experimental series. The five membership functions of the output variable are depicted in Fig. $2 d$ for the first data set. The "yield-strength" variable for the second data set differs only in the range, which is between 107 and 193. The typical number of membership functions for a variable is between three and seven [2, 6, 12, 27, 35], two and more than seven membership functions being a more rare case [4, 12]. The number of five membership functions was chosen for most variables because it allowed for good coverage of the variable ranges from the data sets. Only the coarsenesses of microstructure were measured with three distinct values, which required three membership functions to represent them.

\subsection{Fuzzy inference rules}

The second phase was to derive fuzzy inference rules from the training data sets. The process of the rule creation was carried out by a knowledge engineering expert. Each data point within the training data was inspected, and a collection of IF-THEN rule was formulated using the defined linguistic variables and logical connectives. The process consisted of four steps:

1. All numerical values were replaced with linguistic labels (names of membership functions) and grouped by the second input $(\mathrm{Cu}$ or $\mathrm{Si}$ percentage) due to this input being natural ordering in the data sets.

2. Repetitive rules were removed and rule conflicts were resolved, a conflict being two rules with the same antecedent but implying different consequents. The conflict resolution consisted of selecting the consequent that provided a higher membership value for the given output value (yield strength) from the data point. 
Table 1 Fuzzy linguistic variables and values for each parameter

\begin{tabular}{llc}
\hline Linguistic variable & Linguistic values & Range \\
\hline The first data set & & \\
Inputs & & $10-50$ \\
1: SDAS & Fine, average, coarse & $0-5.5$ \\
2: Cu-amount & Very little, little, average much, very much, \\
Output & Very low, low, average, high, very high & $113-210$ \\
Yield-strength & & \\
The second data set & & \\
Inputs & Fine, average, coarse & $10-50$ \\
1: SDAS & Very little, little, average, much, very much & $7-14.5$ \\
2: Si-amount & & \\
Output & Very low, low, average, high, very high & $107-193$ \\
Yield-strength &
\end{tabular}

(a)

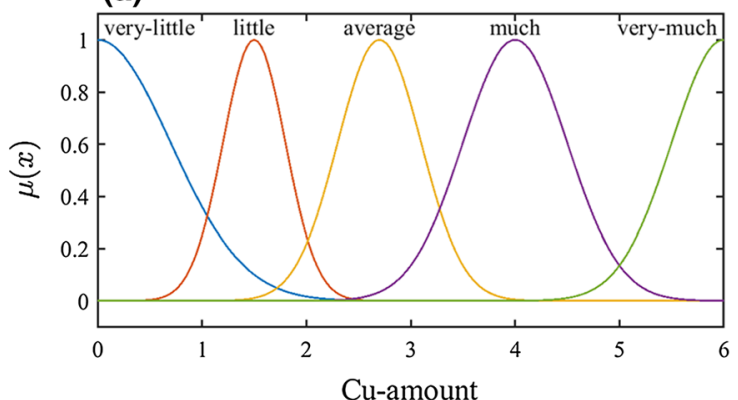

(c)

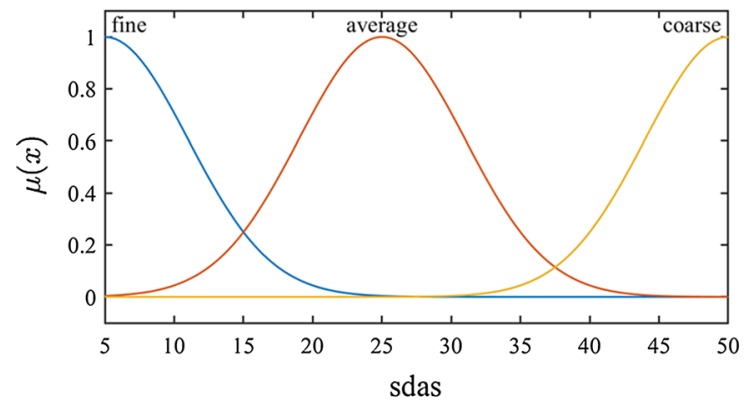

(b)

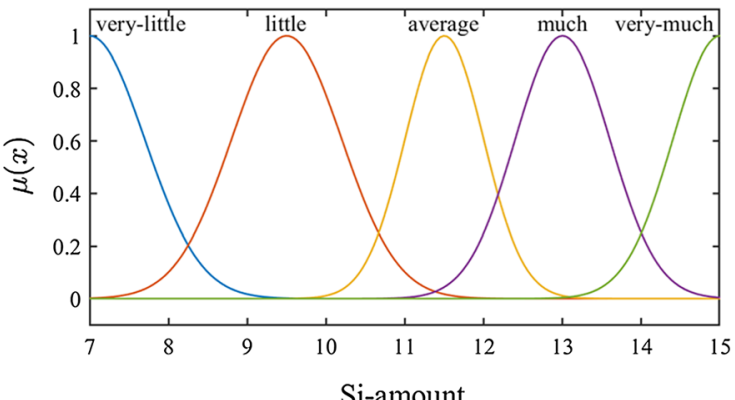

(d)

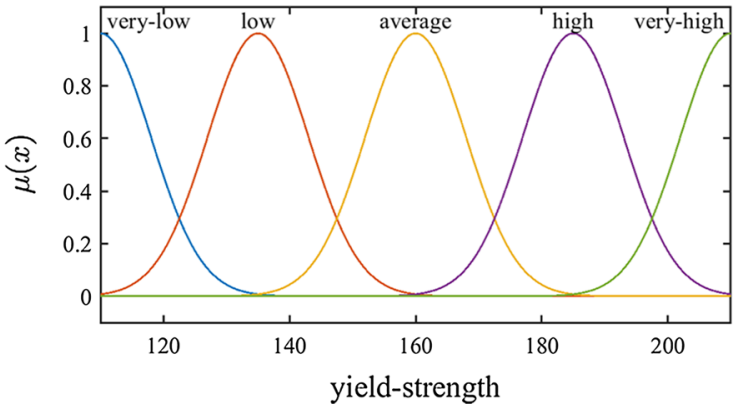

Fig. 2 Membership functions for the input and output parameters. For each function, the $x$-axis indicates input values and the $y$-axis indicates their corresponding degree of membership

3. Rules with the same second input were aggregated by removing the first input (SDAS) when it did not change the consequent in those rules.

4. Rules with the same first input were aggregated by removing the second input when it did not change the consequent in those rules.

The four-step process led to the formalisation of 11 distinct fuzzy rules from the first training data set, which are listed in Table 2 in symbolic format \& denote logical conjunction and implemented as min. The logical implication is also implemented as min. The 10 rules for the second data set are presented in Table 3. The first two rules in both tables specify values of only one variable in the antecedent because the other input variable, that is SDAS, does not affect the values in the consequent.

\section{Evaluation of the accuracy of fuzzy model prediction}

The prediction accuracy of the constructed fuzzy logic systems was evaluated with the help of the test data sets and measured using Mean Absolute Percentage Error (MAPE) [9]. Every pair of input values from each test data set was entered into the corresponding fuzzy logic system 
Table 2 List of fuzzy inference rules derived from the first training data set

\begin{tabular}{|c|c|c|}
\hline Rule no. & Antecedent & Consequent \\
\hline 1 & $(\mathrm{Cu}$-amount==average $)$ & (yield-strength=average) \\
\hline 2 & $(\mathrm{Cu}-\mathrm{amount}==\mathrm{much})$ & (yield-strength=average) \\
\hline 3 & $($ sdas $==$ fine $) \&(\mathrm{Cu}$-amount==very-little $)$ & (yield-strength=low) \\
\hline 4 & $($ sdas $==$ average $) \&(\mathrm{Cu}$-amount==very-little $)$ & (yield-strength=low) \\
\hline 5 & $($ sdas $==$ coarse $) \&($ Cu-amount==very-little $)$ & (yield-strength=very-low) \\
\hline 6 & $($ sdas $==$ fine $) \&(\mathrm{Cu}$-amount $==$ little $)$ & (yield-strength=average) \\
\hline 7 & $($ sdas $==$ average $) \&(\mathrm{Cu}$-amount $==$ little $)$ & (yield-strength=average) \\
\hline 8 & $($ sdas $==$ coarse $) \&(\mathrm{Cu}$-amount==little $)$ & (yield-strength=low) \\
\hline 9 & $($ sdas $==$ fine $) \&(\mathrm{Cu}-$ amount $==$ very-much $)$ & (yield-strength=high) \\
\hline 10 & $($ sdas $==$ average $) \&(\mathrm{Cu}$-amount==very-much $)$ & (yield-strength=very-high) \\
\hline 11 & $($ sdas $==$ coarse $) \&(\mathrm{Cu}-$ amount==very-much $)$ & (yield-strength=high) \\
\hline
\end{tabular}

Table 3 List of fuzzy inference rules derived from the second training data set

\begin{tabular}{|c|c|c|}
\hline Rule no. & Antecedent & Consequent \\
\hline 1 & $($ Si-amount==very-little $)$ & (yield-strength=low) \\
\hline 2 & (sdas $==$ average $)$ & (yield-strength=low) \\
\hline 3 & $($ sdas $==$ fine $) \&($ Si-amount $==$ little $)$ & (yield-strength=average) \\
\hline 4 & $($ sdas $==$ coarse $) \&($ Si-amount $==$ little $)$ & (yield-strength=very-low) \\
\hline 5 & $($ sdas $==$ fine $) \&($ Si-amount $==$ average $)$ & (yield-strength=high) \\
\hline 6 & $($ sdas $==$ coarse $) \&($ Si-amount $==$ average $)$ & (yield-strength=very-low) \\
\hline 7 & $($ sdas $==$ fine $) \&($ Si-amount $==$ much $)$ & (yield-strength=very-high) \\
\hline 8 & $($ sdas $==$ coarse $) \&($ Si-amount $==$ much $)$ & (yield-strength=very-low) \\
\hline 9 & $($ sdas $==$ fine $) \&($ Si-amount==very-much $)$ & (yield-strength=very-high) \\
\hline 10 & $($ sdas $==$ coarse $) \&($ Si-amount==very-much $)$ & (yield-strength=low) \\
\hline
\end{tabular}

to compute an output value. After that, MAPE was computed by dividing the absolute difference of the predicted and measured values by the absolute value of the measured value and averaging this ratio over the data. MAPE was chosen as error measure due to the following reason. Unitfree measures are often preferred because the scale of data can vary significantly and there are many data sets with large numbers [1]. MAPE is frequently used when a unitfree measure is required. Moreover, MAPE offers explication of the accuracy of prediction as a relative error [9]. The disadvantages of MAPE are that it is not applicable when the quantity can take zero values as well as it is unbalanced in terms of giving more penalty for values that are higher than the measured values [1, 9]. In this study, all the experimental and predicted values are nonzero and there is no prevalence of predicted values that significantly exceed the measured values. Thus, MAPE is a suitable error measure that allows for intuitive interpretation of prediction accuracy and comparison of different predictive models.

The evaluation results are presented in Table 4 for the first test data set and in Table 5 for the second test data set. The first column in each table contains the designation of a casting component from the test data sets. Both tables show the percentage of $\mathrm{Cu} / \mathrm{Si}$ and SDAS, which are the input values to the fuzzy logic models, as well as the measured value of yield strength in the next three columns. The values predicted by the fuzzy systems are shown in the fifth column of each table. The last column contains a (signed) percentage error for each pair of predicted and measured values. MAPE is given at the bottom of each table.

The resulting prediction errors, $3.53 \%$ for the first fuzzy logic model and $3.19 \%$ for the second model, are of the same magnitude as the prediction accuracy of the fuzzy logic-based systems reported in the related work described in Sect. 2.2. The prediction errors were also discussed with the domain experts, and they deemed the error level as acceptable. However, the fuzzy logic models were created from small data sets as this is initial work on modelling of yield strength. When more extensive data sets are used for the modelling, the prediction accuracy may change. Additionally, the procedure of data collection at a particular laboratory or foundry could affect the performance of the fuzzy logic models as well.

The percentage prediction errors are additionally depicted in Fig. 3, which plots the relative errors in relation to the measured values of yield strength. The examination of the plot in Fig. $3 \mathrm{~b}$ indicates that the biggest errors are 
Table 4 Evaluation of the error of prediction for the first test data set

\begin{tabular}{lllllr}
\hline Sample & Cu & SDAS & YS measured & YS predicted & Error \\
\hline AA13 & 0 & 10 & 125.44 & 135.02 & 7.64 \\
AA23 & 0 & 20 & 125.51 & 135.02 & 7.58 \\
AA33 & 0 & 50 & 113.06 & 116.09 & 2.68 \\
AB13 & 0.6 & 10 & 133.59 & 135.64 & 1.54 \\
AB22 & 0.6 & 20 & 135.94 & 135.64 & -0.22 \\
AB32 & 0.6 & 50 & 117.57 & 117.71 & 0.12 \\
AC11 & 1 & 10 & 144.96 & 145.58 & 0.43 \\
AC21 & 1 & 20 & 145.11 & 145.58 & 0.32 \\
AC31 & 1 & 50 & 129.44 & 129.21 & -0.18 \\
AD13 & 1.5 & 10 & 144.20 & 155.91 & 8.12 \\
AD23 & 1.5 & 20 & 157.29 & 155.91 & -0.88 \\
AD33 & 1.5 & 50 & 139.01 & 134.97 & -2.91 \\
AE12 & 1.7 & 10 & 151.14 & 157.62 & 4.29 \\
AE32 & 1.7 & 50 & 141.50 & 136.76 & -3.35 \\
AF31 & 2.5 & 50 & 152.20 & 159.83 & 5.01 \\
AG14 & 3.5 & 10 & 166.83 & 160.00 & -4.10 \\
AG23 & 3.5 & 20 & 173.85 & 160.00 & -7.97 \\
AG33 & 3.5 & 50 & 170.86 & 160.00 & -6.36 \\
AI12 & 5.5 & 10 & 194.70 & 184.50 & -5.24 \\
AI23 & 5.5 & 20 & 202.88 & 198.19 & -2.31 \\
AI32 & 5.5 & 50 & 189.74 & 184.31 & -2.86 \\
& & & & MAPE & 3.53 \\
& & & & &
\end{tabular}

Table 5 Evaluation of the error of prediction for the second test data set

\begin{tabular}{lllllr}
\hline Sample & Si & SDAS & YS measured & YS predicted & Error \\
\hline AIA13 & 7 & 10 & 125.44 & 127.60 & 1.72 \\
AIA23 & 7 & 20 & 125.51 & 127.60 & 1.66 \\
AIA33 & 7 & 50 & 113.06 & 127.51 & 12.79 \\
AIB13 & 10 & 10 & 161.20 & 148.66 & -7.78 \\
AIB23 & 10 & 20 & 132.35 & 130.20 & -1.62 \\
AIB33 & 10 & 50 & 108.67 & 110.69 & 1.85 \\
AIC13 & 11.5 & 10 & 168.94 & 168.10 & -0.50 \\
AIC33 & 11.5 & 50 & 115.77 & 110.32 & -4.71 \\
AID13 & 12.5 & 10 & 180.14 & 175.48 & -2.59 \\
AID33 & 12.5 & 50 & 110.40 & 110.85 & 0.41 \\
AIE13 & 13 & 10 & 176.33 & 177.76 & 0.81 \\
AIE23 & 13 & 20 & 136.41 & 131.13 & -3.88 \\
AIE33 & 13 & 50 & 110.99 & 110.60 & -0.35 \\
AIF13 & 14.5 & 10 & 176.03 & 178.13 & 1.20 \\
AIF23 & 14.5 & 20 & 128.38 & 130.70 & 1.80 \\
AIF33 & 14.5 & 50 & 118.65 & 127.33 & 7.31 \\
& & & & MAPE & 3.19 \\
\hline
\end{tabular}

(a)

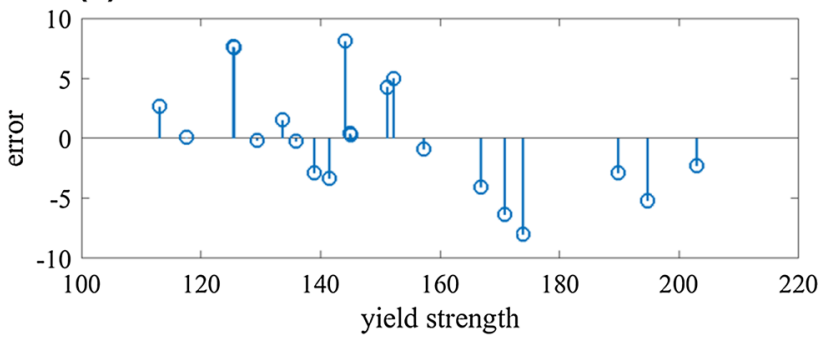

(b)

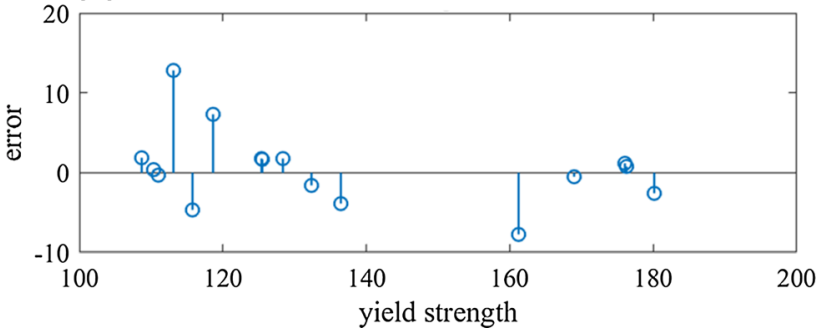

Fig. 3 Percentage errors of the predictions plotted as stems for each value of yield strength: a from the first test data set and $\mathbf{b}$ from the second test data set. The $x$-axis is the baseline containing the measured values

concentrated in the first quarter of the yield strength range. When this plot is compared to the plot of the membership functions for Si-amount in Fig. $2 b$, the error zone of the former corresponds to the first two membership functions: very-little and little. When the values of Si percentage from the second data set were partitioned, these membership functions were assigned only one value each and their bases were made wider than for the other membership functions for Si-amount. Having such "low-density" membership functions is likely the reason for the lower precision in the prediction. There is also one bigger error in the third quarter in Fig. 3b, which might be an outlier because this error is not explained by the membership functions for Si-amount. The plot in Fig. 3a provides less clear indication of big error concentration. The second, third and fourth quarters of the yield strength range contain a cluster of big errors each, which correspond to the membership functions average, much and very-much for $\mathrm{Cu}$-amount in Fig. 2a. The range for $\mathrm{Cu}$ percentage from the first data set was partitioned in the same manner as the range for $\mathrm{Si}$-amount, thus making these three membership functions "low-density" ones and implying the same explanation. The bigger error in the first quarter in Fig. 3a might again be an outlier because this error is not explained by partitioning of the membership functions for $\mathrm{Cu}$ amount. 


\section{Comparison of the fuzzy logic models with ANN prediction approach}

Predictive neural networks have been built based on the Keras neural network sequential model [15] for both data sets. The network architecture and training parameters are presented in Table 6. The ANNs were trained and evaluated using the same groups of training and test data as the ones used for the fuzzy logic models in both data sets. Figure 4 depicts the results obtained with both ANN and fuzzy logic approaches as scatter diagrams. The results indicate that the performances of the two models are similar for the first data set. However, the fuzzy logic model performs better than the ANN model for the second data set. It is not clear why the ANN model performs worse for the second data set. One possible explanation could be that the neural network becomes overfitted due to the small size of the data set, although the sizes of the first and second data sets do not differ much and the network performance is good for the first data set.

\section{Discussion of the results}

Comparison of the prediction errors (MAPE) of the constructed fuzzy logic models $(3.53 \%$ and $3.19 \%)$ with the errors reported in the related work shows that a lower error is reported for the fuzzy logic model for predicting mechanical properties of polyethylene insulation by Boukezzi et al. [4]. It reports Mean Absolute Relative Error (MARE) of 1.6-1.9\% (where MARE is defined in an equivalent manner as MAPE). Polyethylene insulation is a very different material from cast components, and thus, it is difficult to compare the performance of the models. In spite of this, a plausible explanation for this could be that each linguistic variable in [4] is defined by nine membership functions compared to the variables with three and five membership functions in the current study. A finer partitioning of the range of linguistic variables is likely to lead to a better performance of fuzzy inference because it

Table 6 ANN model architecture and training parameters

\begin{tabular}{ll}
\hline The number of hidden layers & 2 \\
The number of hidden units & 10 \\
Activation function & Rectifier linear unit \\
The initial weights & LeCun normal initializer \\
Loss function & Mean squared error \\
Optimizer & Adam \\
Number of iterations & 100 \\
\hline
\end{tabular}

\section{(a)}

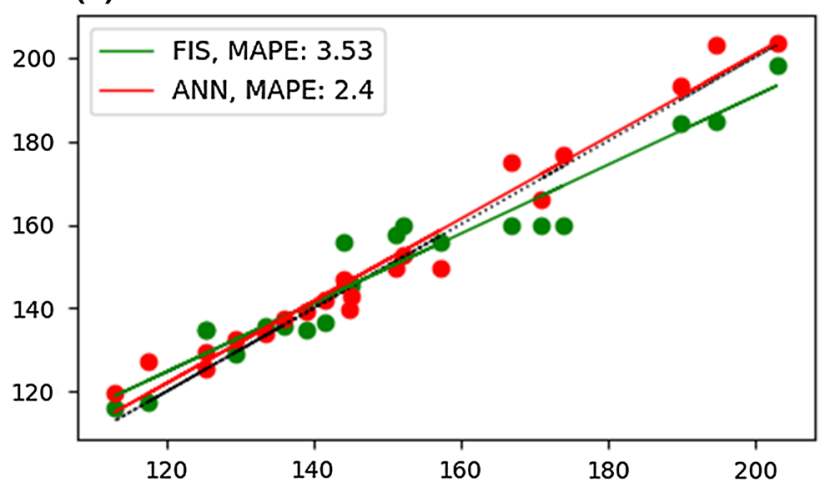

(b)

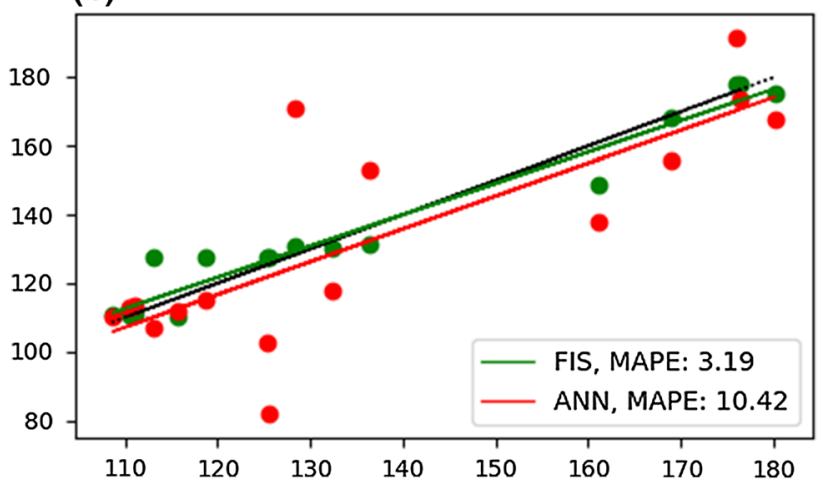

Fig. 4 Comparison of the prediction performance of the fuzzy models and the ANN models based on: $\mathbf{a}$ the first test data set and $\mathbf{b}$ the second test data set. The $x$-axis is the measured value, and the $y$-axis is the predicted value

provides for more accurate mapping of the input-output values. Additionally, the higher number of membership functions usually requires the higher number of fuzzy inference rules. The number of fuzzy rules defined in [4] is 18 , which is higher compared to the current study (11 and 10 rules). Consequently, the higher number of membership functions and the bigger size of the rule set could have contributed to better accuracy reported in [4].

The errors of the fuzzy logic-based systems for prediction of surface hardness/roughness of metallic materials are similar to the errors of the constructed fuzzy logic models: MAPE of $3.85 \%$ is reported by Zalnezhad et al. [35] and MAPE of $5.4 \%$ by Barzani et al. [2]. In these studies, the numbers of membership functions for linguistic variables are not significantly different from the variables in the current work. In [2], two variables have four membership functions and the other two have three membership functions, and in [35] all the variables have four membership functions, which is similar to the current study. One can note that both Zalnezhad et al. [35] and Barzani et al. [2] transformed one data point in a training data set to one inference rule, resulting in 16 rules in [35] and 36 rules in 
[2]. In the current study, a different procedure has been used for rule construction (see Sect. 4.2), which led to smaller sets of rules: 11 rules in the first model and 10 rules in the second model. Having fewer fuzzy inference rules, while keeping the same level of accuracy is advantageous in terms of performance but also improves the interpretability of the constructed fuzzy logic model. However, all the compared studies have been constructed from relatively small data sets and the comparison results may change when more data are used to fine-tune the systems. Additionally, the modelled components have different material characteristics, which further complicates the comparison.

Most related studies described in Sect. 2.2 used the manual approach to build fuzzy logic models and very few details of this construction process are revealed in the reviewed papers. In contrast, an attempt has been made in the current study to present the procedures for manual creation of membership functions (Sect. 4.1) and fuzzy inference rules (Sect. 4.2). Up to now, these procedures have only been used to build two fuzzy logic models, yet with a successful outcome. It is worth to mention one result of applying the rule construction procedure. The first two rules in Tables 2 and 3 specify values of only one variable in the antecedent of those rules as soon as one of the input variables does not affect the values in the consequent. This implies that microstructure coarseness does not have an impact on the results when the percentage of $\mathrm{Cu}$ is average or high for the first type of alloy. In the case of the second alloy type, when the amount of $\mathrm{Si}$ is very low, coarseness does not affect yield strength as well as when the coarseness is average, the Si percentage does not have an impact on the results.

\section{Conclusions}

This paper has proposed a fuzzy logic approach to map the yield strength of an as-cast component to the coarseness of microstructure and the percentage of $\mathrm{Cu} / \mathrm{Si}$ in a cast alloy. Two fuzzy logic models were constructed and evaluated with the help of data obtained during the experimental work on die casting and tensile testing. The same mapping has been also modelled with artificial neural networks and compared to the fuzzy logic results. The evaluation of the fuzzy logic models indicates the prediction errors of $3.53 \%$ for the first model and $3.19 \%$ for the second model. The prediction accuracy of the proposed models is at the same level as the accuracy of the fuzzy logic-based systems for prediction of surface hardness/roughness of metallic materials reviewed in Sect. 2.2. The domain experts considered the error level as acceptable as well. The comparison to the ANN approach shows that the accuracy of the approaches is similar, although the ANN yields a worse result for the second data set. However, the main advantage of fuzzy logic systems over ANN is that the former provides transparent models, while the latter results in opaque models. Having transparent models that can be inspected is important for industrial applications. The presented results address the lack of research into modelling of mechanical properties of as-cast components related to the chemical composition of as-cast A356 alloy type and the parameters of a solidification equipment.

The examination of the prediction accuracy of the constructed models suggests that the partitioning of the range of a linguistic variable may affect the size of the error of prediction. In the experimental data sets, the values of the percentage of $\mathrm{Cu} / \mathrm{Si}$ were not evenly distributed over the range, which led to bigger prediction errors in the regions where the values were scarce. The number of membership functions of linguistic variables might also affect the accuracy of prediction because the higher number of membership functions provides for more accurate mapping of the input-output values. The comparison of the presented results with the work by Boukezzi et al. [4] is likely to indicate that counting on twice the amount of membership functions (nine compared to 3-4) could provide for two times lower prediction error. Though, this comparison was difficult to carry out due to the different nature of the modelled materials.

The paper has also introduced the manual procedure for creation of membership functions and fuzzy inference rules. The specified steps can be used to create fuzzy logic models from empirical data in other domains as well. One outcome of this procedure is that the built models achieved the same level of accuracy with fewer inference rules compared to the work of $[2,35]$, while having the similar amount of membership functions. In general, having fewer inference rules is advantageous because it leads to better performance of a fuzzy logic system and a higher level of interpretability. However, the presented fuzzy logic models have been created from relatively small data sets. The models and the creation procedure need to be further improved and tested with the help of additional experimental data.

Future work will be arranged along the following lines. Firstly, the fuzzy logic models will be extended by incorporating other mechanical properties of cast components. These properties include ultimate tensile strength, elongation to failure and Young's modulus. In addition, more extensive data sets will be used for creation and evaluation. Secondly, methods will be explored for learning fuzzy inference rules and membership function parameters from training data, e.g. the use of genetic algorithms. Manual creation of fuzzy logic models serves the purpose well as demonstrated by this study and several of the papers 
described in Sect. 2.2. However, when the volume of data underlying the model development increases, the manual creation of models becomes unfeasible. The performance of the fuzzy logic models built automatically using the aforementioned methods will be compared to the performance of the models constructed manually as well as built with machine learning methods, e.g. artificial neural networks. Finally, more experimentation is needed to investigate how the partitioning of variable ranges and the number of membership functions affects the prediction accuracy of a fuzzy logic model.

\section{Compliance with ethical standards}

Conflict of interest The authors declare that they have no conflict of interest.

Open Access This article is distributed under the terms of the Creative Commons Attribution 4.0 International License (http://creative commons.org/licenses/by/4.0/), which permits unrestricted use, distribution, and reproduction in any medium, provided you give appropriate credit to the original author(s) and the source, provide a link to the Creative Commons license, and indicate if changes were made.

\section{References}

1. Armstrong J, Collopy F (1992) Error measures for generalizing about forecasting methods: empirical comparisons. Int J Forecast 8(1):69-80. https://doi.org/10.1016/0169-2070(92)90008-W

2. Barzani MM, Zalnezhad E, Sarhan AA, Farahany S, Ramesh S (2015) Fuzzy logic based model for predicting surface roughness of machined $\mathrm{Al}-\mathrm{Si}-\mathrm{Cu}-\mathrm{Fe}$ die casting alloy using different additives-turning. Measurement 61(Supplement C):150-161. https://doi.org/10.1016/j.measurement.2014.10.003

3. Błaszczuk A, Krzywański J (2017) A comparison of fuzzy logic and cluster renewal approaches for heat transfer modeling in a $1296 \mathrm{t} / \mathrm{h}$ CFB boiler with low level of flue gas recirculation. Arch Thermodyn 38(1):91-122. https://doi.org/10.1515/aoter-20170006

4. Boukezzi L, Bessissa L, Boubakeur A, Mahi D (2017) Neural networks and fuzzy logic approaches to predict mechanical properties of XLPE insulation cables under thermal aging. Neural Comput Appl 28(11):3557-3570. https://doi.org/10.1007/s00521016-2259-y

5. Castillo E, Fernández-Canteli A, Hadi AS (1999) On fitting a fatigue model to data. Int J Fatigue 21(1):97-106

6. Chiang KT, Liu NM, Chou CC (2008) Machining parameters optimization on the die casting process of magnesium alloy using the grey-based fuzzy algorithm. Int $\mathrm{J}$ Adv Manuf Technol 38(3):229-237. https://doi.org/10.1007/s00170-007-1103-z

7. Clark W, Hudak S (1975) Variability in fatigue crack growth rate testing. J Test Eval 3(6):454-476

8. Cui W (2002) A state-of-the-art review on fatigue life prediction methods for metal structures. J Mar Sci Technol 7(1):43-56

9. de Myttenaere A, Golden B, Grand BL, Rossi F (2016) Mean absolute percentage error for regression models. Neurocomputing 192:38-48. https://doi.org/10.1016/j.neucom.2015.12.114
10. Haghdadi N, Zarei-Hanzaki A, Khalesian A, Abedi H (2013) Artificial neural network modeling to predict the hot deformation behavior of an a356 aluminum alloy. Mater Des 49:386-391

11. Hasani H, Babaie S (2018) Selfish node detection in ad hoc networks based on fuzzy logic. Neural Comput Appl. https://doi. org/10.1007/s00521-018-3431-3

12. Iqbal A, He N, Li L, Dar NU (2007) A fuzzy expert system for optimizing parameters and predicting performance measures in hard-milling process. Expert Syst Appl 32(4):1020-1027. https:// doi.org/10.1016/j.eswa.2006.02.003

13. Jarfors AEW, Bejhem M, Carlsson T, Eliasson A, Keife H, Nicolescu CM, Rundqvist B (2010) Tillverkningsteknologi, 4th edn. Studentlitteratur, Lund

14. Karaci A (2018) Intelligent tutoring system model based on fuzzy logic and constraint-based student model. Neural Comput Appl. https://doi.org/10.1007/s00521-017-3311-2

15. Keras: The Keras sequential model. https://keras.io/models/ sequential/

16. Krupp U (2007) Fatigue crack propagation in metals and alloys: microstructural aspects and modelling concepts. Wiley, London

17. Krzywanski J, Wesolowska M, Blaszczuk A, Majchrzak A, Komorowski M, Nowak W (2016) The non-iterative estimation of bed-to-wall heat transfer coefficient in a CFBC by fuzzy logic methods. Procedia Eng 157:66-71. https://doi.org/10.1016/j. proeng.2016.08.339

18. Krzywanski J, Wesolowska M, Blaszczuk A, Majchrzak A, Komorowski M, Nowak W (2018) Fuzzy logic and bed-to-wall heat transfer in a large-scale CFBC. Int J Numer Methods Heat Fluid Flow 28(1):254-266. https://doi.org/10.1108/hff-09-20170357

19. Liu Y, Mahadevan S (2009) Probabilistic fatigue life prediction using an equivalent initial flaw size distribution. Int $\mathbf{J}$ Fatigue 31(3):476-487

20. Lumley RN, Deeva N, Larsen R, Gembarovic J, Freeman J (2013) The role of alloy composition and T7 heat treatment in enhancing thermal conductivity of aluminum high pressure diecastings. Metall Mater Trans A 44(2):1074-1086. https://doi. org/10.1007/s11661-012-1443-7

21. Malinov S, Sha W, McKeown J (2001) Modelling the correlation between processing parameters and properties in titanium alloys using artificial neural network. Comput Mater Sci 21(3):375-394

22. Nasrollahzadeh K, Afzali S (2018) Fuzzy logic model for pullout capacity of near-surface-mounted FRP reinforcement bonded to concrete. Neural Comput Appl. https://doi.org/10.1007/s00521018-3590-2

23. Ozerdem MS, Kolukisa S (2009) Artificial neural network approach to predict the mechanical properties of $\mathrm{Cu}-\mathrm{Sn}-\mathrm{Pb}-\mathrm{Zn}-$ Ni cast alloys. Mater Des 30(3):764-769

24. Precup RE, Hellendoorn H (2011) A survey on industrial applications of fuzzy control. Comput Ind 62(3):213-226

25. Raza M, Svenningsson R, Irwin M, Fägerström B, Jarfors AE (2017) Effects of process related variations on fillablity simulation of thin-walled IN718 structures. Springer, Berlin

26. Schaffer J (2006) A probabilistic approach to modeling microstructural variability and fatigue behavior in ASTM F562 medical grade wire. In: Proceedings of the 9th international congress on fatigue, fatigue 2006

27. Shabani MO, Rahimipour MR, Tofigh AA, Davami P (2015) Refined microstructure of compo cast nanocomposites: the performance of combined neuro-computing, fuzzy logic and particle swarm techniques. Neural Comput Appl 26(4):899-909. https:// doi.org/10.1007/s00521-014-1724-8

28. Shirani M, Härkegård G (2012) Damage tolerant design of cast components based on defects detected by 3D X-ray computed tomography. Int J Fatigue 41(Supplement C):188-198. https:// doi.org/10.1016/j.ijfatigue.2011.09.011 
29. Sigworth $G$ (2011) Understanding quality in aluminum castings. Int J Metalcast 5(1):7-22. https://doi.org/10.1007/BF03355504

30. Wang Q (2003) Microstructural effects on the tensile and fracture behavior of aluminum casting alloys A356/357. Metall Mater Trans A 34(12):2887-2899

31. Weibull W et al (1951) A statistical distribution function of wide applicability. J Appl Mech 18(3):293-297

32. Wu W, Ni C (2004) Probabilistic models of fatigue crack propagation and their experimental verification. Probab Eng Mech 19(3):247-257

33. Zadeh LA (1986) Is probability theory sufficient for dealing with uncertainty in AI: a negative view. In: Machine intelligence and pattern recognition, vol 4. Elsevier, Amsterdam, pp 103-116
34. Zadeh LA (1973) Outline of a new approach to the analysis of complex systems and decision processes. IEEE Trans Syst Man Cybern SMC-3(1):28-44. https://doi.org/10.1109/TSMC.1973. 5408575

35. Zalnezhad E, Sarhan AAD, Hamdi M (2013) A fuzzy logic based model to predict surface hardness of thin film TiN coating on aerospace AL7075-T6 alloy. Int J Adv Manuf Technol 68(1):415-423. https://doi.org/10.1007/s00170-013-4738-y

Publisher's Note Springer Nature remains neutral with regard to jurisdictional claims in published maps and institutional affiliations. 